\title{
Use of Data Mining Technique for Prediction of Crop Disease \& Yield in The Face of Climate Change and Market Analysis Strategy
} Pallavi Chature ${ }^{1}$, Pallavi Borde ${ }^{2}$, Rohini Khemnar ${ }^{3}$, Sonal Dhokane ${ }^{4}$, Prof. N.B. Kadu ${ }^{5}$

${ }^{1-4}$ Students, Department of Information Technology, SPPU, Pune, Maharashtra, India ${ }^{5}$ Assistant Professor, Department of Information Technology, SPPU, Pune, Maharashtra, India

\begin{abstract}
Data mining and machine gaining knowledge of is an emerging field of research in facts era in addition to in agriculture. Agrarian sector is facing rigorous trouble to maximize the crop productiveness. The present have a look at makes a specialty of the packages of data mining strategies in crop sickness prediction in the face of climatic trade to assist the farmer in taking choice for farming and accomplishing the predicted monetary go back. The Crop disease prediction is a prime hassle that may be solved based totally on available data. Data mining strategies are the better selections for this purpose. Exclusive data mining techniques are used and evaluated in agriculture for estimating the future year's crop production. The main cause of the gadget is for social use. Farmer has to face many troubles like lack of know-how, Manures, fertilizers and Agriculture marketing etc. gift method SAR Tomography takes the photographs and gives the exceptional development stages of crop. This system not gives the fertilizers and precautions to the farmer. This paper gives quick analysis of crop disease prediction the usage of $\mathrm{k}$ Nearest Neighbour class approach and Density based clustering approach for the chosen place. The styles of crop production in response to the climatic (rainfall, temperature, relative humidity and sunshine) impact across the selected regions are being evolved using ok Nearest Neighbour technique. For that reason, it is going to be useful if farmers should use the technique to are expecting the future crop productivity and therefore adopt opportunity adaptive measures to maximize yield if the predictions fall below expectations and business viability.
\end{abstract}

Keywords : Data mining, Machine Learning, Classification, Clustering, Market Survey, Weather Prediction Techniques, API, Crop Disease Prediction, Recommendations of Fertilizers, Precautions

\section{INTRODUCTION}

Crop prediction is the art of predicting crop yields and manufacturing earlier than the harvest virtually takes area, usually more than one month earlier. Crop forecasting is based on computer programs that describe the plant surroundings interactions in quantitative terms. The soil testing program starts with the collection of a soil sample from afield. the primary principle of soil testing is that a discipline may be sampled in such a way that chemical evaluation of the soil pattern will correctly reflect the sphere's authentic nutrient fame. The motive of soil trying out in excessiveyield farming is to decide the relative potential of a soil to supply crop nutrients all through a selected growing season, to decide the wishes, and for diagnosing issues which include excessive salinity or alkalinity. Soil checking out is likewise used to manual nutrient management choices related to 
manure and sludge utility with the objective of maximizing economic/agronomic benefits whilst minimizing the ability for negative effects on water fine. Data mining is a technique of extracting hidden information from a database and transforms it into an understandable shape for further use. Its miles the computational technique of coming across styles in massive information sets related to strategies at the intersection of synthetic intelligence, machine learning, records, and database systems. The remaining intention of data mining is prediction - and predictive records mining is the most commonplace form of information mining and one that has the maximum direct commercial enterprise programs. For the duration of the years, many algorithms have been created to extract know-how from huge units of statistics. There are numerous distinctive methodologies to approach this problem: category, affiliation rule, clustering, etc. right here we are able to awareness on category technique. Class strategies are designed for classifying unknown samples the usage of information provided by using a fixed of categorized samples.

This set is normally referred to as a training set, due to the fact, in well-known; it is used to teach the category approach how to perform its category. The class venture may be visible as a supervised technique in which each example belongs to a class, that is indicated by means of the value of a unique aim characteristic or sincerely the elegance attributes. type exercises with statistics mining use a diffusion of algorithms and the precise set of rules used can affect the manner records are categorized. This work talks approximately choice Tree classifier assumes that the presence (or absence) of a particular function of a class is unrelated to the presence (or absence) of another feature. Depending on the precise nature of the probability version, ok Nearest Neighbour $(\mathrm{kNN})$ and Density based totally clustering can be skilled very successfully in a supervised learning placing.

\section{PROBLEM STATEMENT}

\section{Problem Statement:}

Data mining is an emerging field of research in Information Technology as well as in agriculture. Agrarian sector in India is facing rigorous problem to maximize the crop productivity. The present study focuses on the applications of data mining techniques in yield prediction in the face of climatic change to help the farmer in taking decision for farming and achieving the expected economic return. The problem of yield as well as disease prediction is a major problem that can be solved based on available data. Hence, we proposed a system Prediction of "Use of Data Mining Technique for Prediction of Crop Yield in The Face of Climate Change and Market Analysis Strategy".

\section{Goals \& Objectives:}

- To predict whether before sowing crop.

- Prediction the disease before the sowing crop (using the weather condition)

- To provide precaution and fertilizer recommendation.

- To analyse the disease at post condition using SIFT algorithm.

- To reduce productivity/production cost and increase income.

- To reduce fertilizers on crop.

\section{LITERATURE REVIEW}

Rupanjali D. Baruah, Sudipta Roy, R.M. Bhagat, L.N. Sethi "Use of Data Mining Technique for Prediction of Tea Yield in the Face of Climate 
Change of Assam, India", 2016 International Conference on Information Technology.

This paper presents an analysis using data mining techniques for estimating the future yield prediction in tea cultivation with climatic change trends observed in last 30 years (1977-2006). The patterns of crop production in response to the climatic (rainfall, temperature, relative humidity, evaporation and sunshine) effect across the four tea growing regions (South Bank, North Bank, Upper Assam and Cachar) of Assam were developed using Multiple Linear Regression (MLR) technique. The tea production estimation equations developed for the regions were validated for the future yield prediction (2007, 2009 and 2010) and were found to be significant. Thus, it is suggested that the planters/farmers could use the technique to predict the future crop productivity and consequently adopt alternative adaptive measures to maximize yield if the predictions fall below expectations and commercial viability.

Gregory S. McMaster, DA Edmunds, W.W. Wilhelm, 1, D.C. Nielsen, P.v.v. Prasad.c. Ascough, "PhenologyMMS: The objective of this paper is to provide an overview of a decision support technology software tool, Phenology MMS Vl.2, developed to simulate the phenology of various crops for varying levels of soil water. The program is intended to be simple to use, requires minimal information for calibration, and can be incorporated into other crop simulation models. It consists of a Java interface connected to FORTRAN science modules to simulate phenological responses. The complete developmental sequence of the shoot apex correlated with phenological events, and the response to soil water availability for winter and spring wheat and sunflower were created based on experimental data and the literature. Model evaluation consisted of testing algorithms using "generic" default phenology parameters for wheat (i.e., no calibration for specific cultivars was used) for a variety of field experiments to predict developmental events. Results demonstrated that the program has general applicability for predicting crop phenology and can aid in crop management.

Bruno Basso, Davide Cammarano, Elisabetta Carfagna, Review of Crop Yield Forecasting Methods and Early Warning Systems", Journal of convergence in engineering, technology and science, Vol.1, pp.1-8,2009. The following review paper presents an overview of the current crop yield forecasting methods and early warning systems for the global strategy to improve agricultural and rural statistics across the globe. Different sections describing simulation models, remote sensing, yield gap analysis, and methods to yield forecasting compose the manuscript.

Young Ju Jeong, Kwang Eun An, Sung Won Lee, and Dongmahn Seo, "Improved Durability of Soil Humidity Sensor for Agricultural IoT environments", 2018 IEEE International Conference on Consumer Electronics (ICCE) Soil humidity is the most important factor for plant growth. Therefore, the soil humidity sensor is an important part of smart farm application using agricultural IoT environments. Since soil humidity sensors are applied wet underground and the sensor consists of copper, rust eats away the copper surface of sensors. From rusting of sensors, wrong information of soil humidity can be collected on smart farm system based on agricultural IoT Environments. It makes that smart farm is not reliable. In this paper, we propose a new type of soil humidity sensor in order to extend life time.

M. Trnka, M. Dubrovsky, D. Semeradova, and Z. Z alud, Projections of uncertainties in climate change 
scenarios into expected winter wheat yields", in Proceedings of the 11th European Conference on Computer Vision: Part I, pp. 285298,2003. The crop model CERES-Wheat in combination with the stochastic weather generator were used to quantify the effect of uncertainties in selected climate change scenarios on the yields of winter wheat, which is the most important European cereal crop. Seven experimental sites with the high-quality experimental data were selected in order to evaluate the crop model and to carry out the climate change impact analysis. The analysis was based on the multiyear crop model simulations run with the daily weather series prepared by the stochastic weather generator. Seven global circulation models (GCMs) were used to derive the climate change scenarios. In addition, seven GCMbased scenariosm were averaged in order to derive the average scenario (AVG).

\section{PROPOSED SYSTEM}

The preparation of soil is step one before developing a crop. One of the maximum crucial responsibilities in agricultural is to show the soil and unfasten it. This permits the roots to penetrate deep into the soil. The loose soil permits the roots to respire effortlessly even if they go deep into the soil. The loosened soil facilitates in the increase of earthworm and microbes' gift inside the soil. Those organisms are pals of the farmer when you consider that they in addition turn and free the soil and upload humus to it.

Here we're the usage of sensors values like soil moisture sensor, temp sensor, humidity. In this work the experiments are done crucial and widely known type algorithms ok Nearest Neighbour (kNN) and Density based clustering are implemented to the dataset. There accuracy is acquired by comparing the datasets. Every set of rules has been run over the training dataset and their overall performance in phrases of accuracy is evaluated together with the prediction executed inside the trying out dataset. The entire analysis method creates a fact go with the flow.

\section{SYSTEM ARCHITECTURE OVERVIEW}

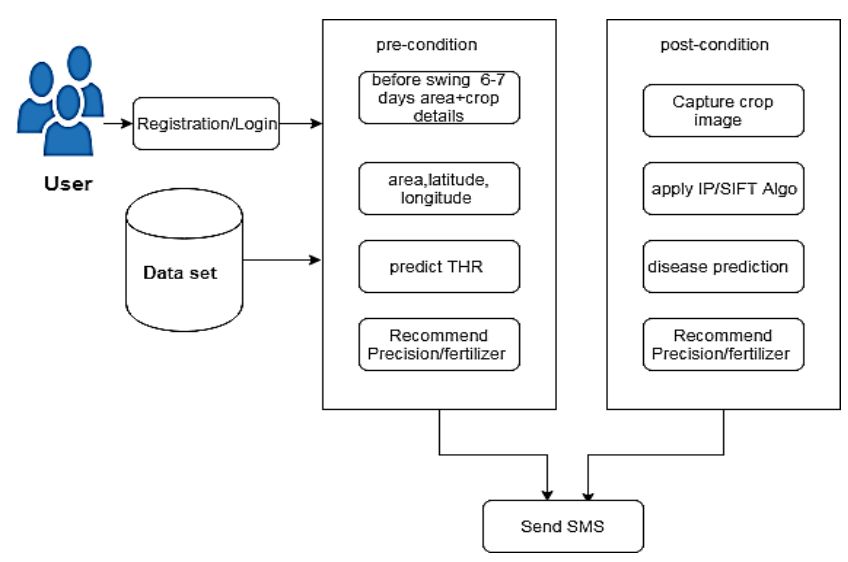

Fig.1 (System Architecture)

\section{RESULTS AND DISCUSSION}

Input:

Here, Whole System taken many more attributes for the input purpose but here author mainly focuses on the Time and performance of system. Based some few attributes we will getting following analytical result for our proposed system.

\section{Performance Measures Used Expected Result:}

\begin{tabular}{|l|l|l|}
\hline Parameter & Existing & Proposed \\
\hline A & 10 & 4 \\
\hline B & 10 & 5 \\
\hline C & 8 & 8 \\
\hline D & 10 & 3 \\
\hline E & 8 & 2 \\
\hline
\end{tabular}

Table.1 Result Table 
$\mathrm{A}=$ Computation Cost.

$\mathrm{B}=$ Time Consumption.

$\mathrm{C}=$ Scalable .

$\mathrm{D}=$ Waiting Time.

$\mathrm{E}=$ User Friendly.

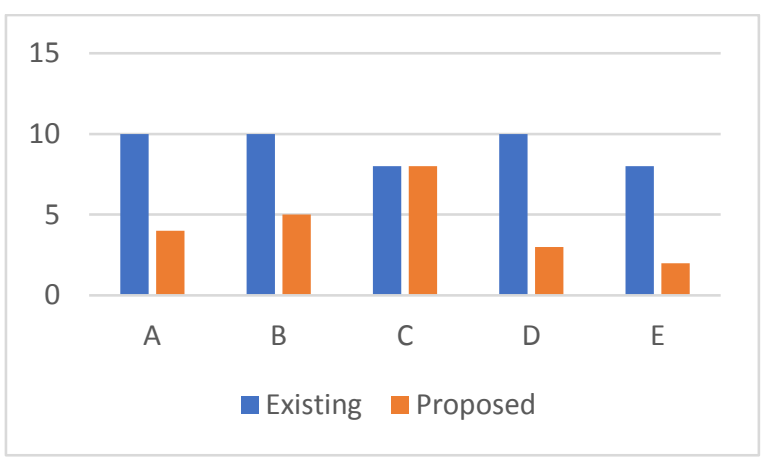

Table. 2 Time line chart of Result Analysis

\section{CONCLUSION AND FUTURE WORK}

This machine focuses on developing automated leaf sicknesses. It saves time and effort, on this undertaking; we've got proposed method for prediction of crop ailment from modern-day weather the use of Google API with the help of $k$ NN set of rules and measuring the crop sicknesses of the crop item and locate climate prediction.

In this work the experiments are achieved two essential and widely known category algorithms kNearest Neighbour (k-NN) and Density primarily based clustering are applied to the dataset. There accuracy is received through evaluating the datasets. Every set of rules has been run over the schooling dataset and their performance in terms of accuracy is evaluated at the side of the prediction completed in the trying out dataset. The complete analysis procedure creates a data flow.

\section{VII.ACKNOWLEDEGMENT}

To develop a Disease Prediction as per Weather Condition and Market Analysis that accurately updates databases according to the weights of goods and maintains transparency in the system and prevents forgery and exploitation of masses caused by consumer.

\section{REFERENCES}

[1]. Adams, R., Fleming, R., Chang, C., McCarl, B., and Rosenzweig, 1993 - A Reassessment of the Economic Effects of Global Climate Change on U.S. Agriculture, Unpublished: September.

[2]. Adams, R., Glyer, D., and McCarl, B. 1989. "The Economic Effects of Climate Change on U. S. Agriculture: A Preliminary Assessment." In Smith, J., and Tirpak, D.,eds., The Potential Effects of Global Climate Change on the United States. Washington, D.C.: USEPA.

[3]. Adams, R.,Rosenzweig, C., Peart, R., Ritchie, J., McCarl,B., Glyer, D., Curry, B., Jones, J., Boote, K., and Allen, H.1990."Global Climate Change and U. S. Agriculture."Nature.345 (6272, May): 219-224.

[4]. Adaptation to Climate Change Issues of Longrun Sustainability." An Economic Research

[5]. Barron, E. J. 1995."Advances in Predicting Global Warming. The Bridge (National Academy of Engineering). 25 (2, Summer): 1015.

[6]. Barua, D. N. 2008. Science and Practice in Tea Culture, second ed. Tea Research Association, Calcutta-Jorhat, India.

[7]. Basu, Majumder, A., Bera, B. and Rajan, A. 2010. Teastatistics: Global scenario. Int. J. Tea Sci.8: 121-124.

[8]. Bazzaz, A., and Fajer, E. D. 1992. "Plant Life in a CO2Rich World. "Scientific American. 1821.

[9]. Brack, D. and M. Grubb. 1996. Climate Change, "A Summary of the Second Assessment Report of the IPCC."FEEM (Fondazione ENI Enrico Mattei, Milano Italy) newsletter, 3, 1996 
[10]. M. Soundarya, R. Balakrishnan," Survey on Classification Techniques in Data mining", International Journal of Advanced Research in Computer and Communication Engineering Vol. 3, Issue 7, July 2014.

[11]. D Ramesh, B Vishnu Vardhan, "Data mining technique and applications to agriculture yield data”, International Journal of Advanced Research in Computer and Communication Engineering Vol. 2, Issue 9, September 2013.

[12]. Gideon O Adeoye, Akinola A Agboola, "Critical levels for soil $\mathrm{pH}$, available $\mathrm{P}, \mathrm{K}, \mathrm{Zn}$ and $\mathrm{Mn}$ and maize ear-leaf content of $\mathrm{P}, \mathrm{Cu}$ and $\mathrm{Mn}$ in sedimentary soils of South- Western Nigeria", Nutrient Cycling in Agroeco systems, Volume 6, Issue 1, pp 65-71, February 1985.

[13]. D. Almaliotis, D. Velemis, S. Bladenopoulou, N. Karapetsas, "Appricot yield in relation to leaf nutrient levels in Northern Greece", ISHS ActaHorticulturae 701: XII International Symposium on Apricot Culture and Decline.

\section{Cite this article as :}

Pallavi Chature, Pallavi Borde, Rohini Khemnar, Sonal Dhokane, Prof. N.B. Kadu, "Use of Data Mining Technique for Prediction of Crop Disease \& Yield in The Face of Climate Change and Market Analysis Strategy", International Journal of Scientific Research in Science and Technology (IJSRST), Online ISSN : 2395-602X, Print ISSN : 2395-6011, Volume 6 Issue 3, pp. 126-131, May-June 2019. Available at doi : https://doi.org/10.32628/IJSRST196312 Journal URL : http://ijsrst.com/IJSRST196312 